\title{
Study on the therapeutic effect of floating needle therapy combined with pressing acupoint embedding for female stress urinary incontinence after childbirth: a randomized trial
}

\author{
Fengna Chen ${ }^{1}$, Jianping Zhou ${ }^{2}$, Weiming $\mathrm{Wu}^{3}$, Xuequn Qian ${ }^{2}$ \\ ${ }^{1}$ Department of Surgery, Ningbo Municipal Hospital of TCM Affiliated Hospital of Zhejiang Chinese Medicine University, Ningbo, China; \\ ${ }^{2}$ Department of Nursing, Ningbo Municipal Hospital of TCM Affiliated Hospital of Zhejiang Chinese Medicine University, Ningbo, China; \\ ${ }^{3}$ Department of Anorectal Surgery, Affiliated Hospital of Medical School of Ningbo University, Ningbo, China \\ Contributions: (I) Conception and design: F Chen; (II) Administrative support: J Zhou; (III) Provision of study materials or patients: W Wu; (IV) \\ Collection and assembly of data: All authors; (V) Data analysis and interpretation: F Chen, X Qian; (VI) Manuscript writing: All authors; (VII) Final \\ approval of manuscript: All authors. \\ Correspondence to: Jianping Zhou. Ningbo Municipal Hospital of TCM Affiliated Hospital of Zhejiang Chinese Medicine University, 819 Liyuan \\ North Road, Haishu District, Ningbo 315010, China. Email: nbszyy30610@163.com.
}

\begin{abstract}
Background Stress urinary incontinence is a common and widespread problem among women that can significantly reduce the quality of life of those affected and potentially result in limitations to the lives and activities of their friends and family members. We aimed to observe the effect of floating needle therapy combined with acupuncture point embedding in the treatment of female stress urinary incontinence after childbirth.
\end{abstract}

Methods: A total of 64 postpartum female stress urinary incontinence patients who were treated and hospitalized in the Urology Clinic of the Ningbo Traditional Chinese Medicine Hospital of the Zhejiang Province from September 2020 to August 2021 were randomly allocated to the experimental group and the control group, each with 32 cases. The control group was treated with the drug midodrine combined with Kegel training, and the experimental group was treated with floating needle therapy combined with acupoint embedding. After 8 weeks of continuous treatment, the pelvic floor muscle strength, urinary incontinence score, and the improvement of urine leakage in the $1 \mathrm{~h}$ pad test were observed. We then evaluated the clinical efficacy of the 2 groups and the state of urinary incontinence at 3 months and 6 months as assessed by telephone follow-up.

Results: After treatment, the pelvic floor muscle strength of the 2 groups had significantly increased, and both the urinary incontinence score and the urine leakage volume of the $1 \mathrm{~h}$ pad test were significantly reduced. The pelvic floor muscle strength of the experimental group was significantly higher than that of the control group after treatment, the urinary incontinence score and the amount of urine leakage of the $1 \mathrm{~h}$ pad test were significantly lower than those of the control group. The total effective rate of the experimental group was $90.63 \%$ (27/32), which was higher than that of the control group (71.88\%, 23/32).

Conclusions: With the benefits of being both convenient and efficient, floating needle therapy combined with press-needle acupoint embedding is effective in treating female stress urinary incontinence after childbirth.

Trial registration: Chinese Clinical Trial Registry ChiCTR2100047558.

Keywords: Stress urinary incontinence; floating needle therapy; press-needle acupoint embedding; curative effect evaluation

Submitted May 07, 2021. Accepted for publication Jul 02, 2021.

doi: 10.21037/apm-21-1382

View this article at: https://dx.doi.org/10.21037/apm-21-1382 


\section{Introduction}

Stress urinary incontinence (SUI) is a condition characterized by an involuntary loss of urine in response to an increase in intra-abdominal pressure due to effort, exertion, sneezing, or coughing. It is a common urological disease in middle-aged and elderly women, known as one of the 5 chronic diseases that compromise women's health (1-3). According to epidemiological investigations, more than $50 \%$ of the incidence of SUI is among older women, and it seriously affects their physical and mental health and social interpersonal communication (4-6). At present, the common clinical treatment of SUI with traditional Chinese medicine (TCM) has many side effects; many women choose to engage in pelvic floor muscle training or Kegel exercises, but it is difficult to master the exercises correctly $(7,8)$. Although many patients are accepting of traditional acupuncture, its effects have been shown to emerge slowly in the treatment of SUI (9). Biofeedback therapy devices are currently used in clinical practice, and a study revealed significant effects on moderate urinary incontinence after 3 courses of treatment (10). Patients with severe SUI treated with tension-free urethral suspension will undoubtedly experience increased physical and mental distress as well as financial burden $(11,12)$, which limits the applicability of this intervention. With this in mind, we treated SUI 64 patients with a new type of therapy based on pelvic floor muscle strength, urinary incontinence score, and $1 \mathrm{~h}$ urine test leakage. We aimed to explore its clinical efficacy, and find new and effective approaches to the clinical treatment of SUI.

We present the following article in accordance with the CONSORT reporting checklist (available at https://dx.doi. org/10.21037/apm-21-1382).

\section{Methods}

\section{Source of information}

From September 2020 to August 2021, 64 female SUI patients were included in the outpatient and inpatient treatment of Ningbo Municipal Hospital of TCM Affiliated Hospital of Zhejiang Chinese Medicine University. According to the random number table method, the participants were allocated to 2 groups, with each group containing 32 cases. All procedures performed in this study involving human participants were in accordance with the Declaration of Helsinki (as revised in 2013). The study was approved by the Ethics Committee of Ningbo Municipal
Hospital of TCM Affiliated Hospital of Zhejiang Chinese Medicine University (AF/SG-0110.1/20201215005) and informed consent was provided by all participants.

\section{Inclusion and exclusion criteria}

The inclusion criteria were as follows: (I) symptoms met the diagnostic criteria for SUI proposed by the International Urinary Inhibition Advisory Board (International Continence Society, ICS) of the International Urinary Control Association, which stipulates that involuntary leakage of urine from the external urethral orifice is induced by sneezing, coughing, and other activities which increase abdominal pressure: (i) Urinary incontinence was deemed to be mild SUI if it was induced when the patient coughed, sneezed, or otherwise increased their abdominal pressure. (ii) Urinary incontinence was classified as moderate SUI if it was induced when patients performed daily activities such as walking and standing up from a chair. (iii) Urinary incontinence was classed as severe SUI if it was noticed during either standing or rest. (II) Urinary incontinence was confirmed by urodynamic tests; no age limit; a history of pregnancy and childbirth; complete clinical data; history of anuria before birth; ability to continue treatment until the end of the course and complete timely follow-up. (III) Participants and their families were fully informed, were willing to voluntarily participate, and provided written informed consent.

The exclusion criteria were as follows: (I) patients with pelvic floor dysfunction and severe pregnancy complications; (II) previous history of urinary incontinence and pelvic organ prolapse; (III) persons with acute inflammation of the urinary system; (IV) the presence of lower urinary tract obstruction; (V) complicating serious primary diseases such as those affecting the cardiovascular, cerebrovascular, liver, kidney, and hematopoietic systems; (VI) increased abdominal pressure due to chronic obstructive emphysema or constipation; (VII) history of vaginitis, pelvic inflammation and urinary tract infection, stones, urinary incontinence; (VIII) urge urinary incontinence and mixed urinary incontinence; (IX) persons with mental illness, cognitive impairment, or intellectual disability.

\section{Treatment measures}

The control group was treated with the drug midodrine in combination with Kegel exercises: the patients assumed a supine position with their legs slightly bent and the whole 
body relaxed. They then inserted 2 fingers into the vagina, contracted the anus and vagina, and relaxed the muscles slowly after $3 \mathrm{~s}$. The process was repeated after a 3-5 s interval. If necessary, the patients' family member was recruited to assist the patient in mastering the technique and removing their fingers. During the exercises, patients were instructed to adjust their breathing, inhaling deeply when they contracted their muscles, exhaling deeply when they relaxed, and concentrating on the feeling of muscular contraction. Participants were instructed to perform 1 Kegel exercise each morning and evening for $15 \mathrm{~min}$, for a total of 8 weeks.

The experimental group was treated with floating acupuncture combined with acupuncture point embedding acupuncture. The floating needle therapy was administered as follows: Disposable floating needles were applied about 4-5 cm from the local myofascial trigger point (MTrP) of the affected muscle, including the inferior segment of the rectus abdominis, oblique abdominal muscle, adductor muscle group of the thigh, medial group of the quadriceps femoris, pelvic floor muscle, and so on. The needles were inserted at a $10-15^{\circ}$ angle into the subcutaneous loose connective tissue, manipulated within about $30^{\circ}$, with a frequency of approximately 100 twists per min, for a total of 2 minutes. At the same time, according to the specifics of the disease and the patient's ability to perform reperfusion activities, the patient would then contract the affected muscle for $10 \mathrm{~s}$, with a 1-2 min interval of rest, and repeat the whole process of scavenging and reperfusion 2-3 times. The needle was then withdrawn, subcutaneous lavage was performed, and the area was fixed with medical tape. Each session of treatment was concluded after $4 \mathrm{~h}$, with 4 sessions treatments performed during week 1, 2 sessions during weeks $2-4$, and 1 session per week during weeks 5-8 for the consolidation period. The pressing acupoint embedding was performed as follows: after the floating needle had been removed and perfused, a $0.2 \mathrm{~mm} \times 1.2 \mathrm{~mm}$ press tack needle was applied to the acupuncture points Zhongji and Guanyuan (4 and 3 anatomical inches below the umbilicus, respectively) with fixed retention for $48 \mathrm{~h}$. The patient was instructed to press the imbedded tack for 1 min each time, 3 times a day during weeks $1-4$, then twice a week during weeks 5-8, and once a week for the consolidation period. A course of treatment lasted for 4 weeks, and participants received continuous intervention for 8 weeks.

\section{Effectiveness evaluation}

The criteria for efficacy were established with reference to the International urinary incontinence advisory committee.

\section{Pelvic floor muscle}

Pelvic floor muscle strength was assessed by the same evaluator before and after treatment with reference to the perineal muscle test. There were 6 potential grades of muscle strength: 0, participants' finger could not feel the contraction of muscle when touching with hand; 1, fingers felt a slight contraction, which could last only $1 \mathrm{~s}$, and this was able to be completed just once; 2 , muscle contraction was obviously felt by the fingers, could be sustained for $2 \mathrm{~s}$, and 2 contractions were able to be completed; 3 , muscle contraction that caused the fingers to move up or forward, action could last $3 \mathrm{~s}$, and could be completed 3 times in a row; 4 , muscular contraction and resistance of pressure from fingers, could last $4 \mathrm{~s}$, and be completed 4 times continuously; and 5, muscular contraction, continuous resistance of finger pressure for up to $5 \mathrm{~s}$ or more, and could be completed more than 5 times. Muscle strength rated as $\leq$ grade 3 was classified as abnormal muscle strength.

\section{Urinary incontinence score}

An international urinary incontinence advisory board urinary incontinence questionnaire (IC-IQ-SF) was used to score urinary incontinence, including 3 dimensions: the frequency of urinary leakage (score 0-5), amount of urinary leakage (score 0-6), and the effect of urinary incontinence on quality of life (score $0-10)$. The total urinary incontinence score was $0-21$. The higher the score of each item, the more serious the symptoms were.

\section{$1 \mathrm{~h}$ urine pad test}

The weight of the women was recorded before the test, and after voiding their bladders, the participants wore adult diapers. Then, they were asked to drink $500 \mathrm{~mL}$ of mineral water in $10 \mathrm{~min}$, rest for 30-50 min, and then commence activities such as squatting up and down, running in situ, stooping to pick up an item, cold water absorption and forced cough, laughter, and so on. After these activities the diapers were removed, weighed again, and the findings were recorded in detail. A weight difference of $2 \mathrm{~g}$ and below was classed as mild, more than $2 \mathrm{~g}$ and less than $10 \mathrm{~g}$ was moderate, more than 10 and less than $50 \mathrm{~g}$ was severe, and $50 \mathrm{~g}$ and above was extremely severe SUI.

\section{Statistical analysis}

Data which were in accordance with normal distribution 
Table 1 Comparison of general information of the 2 groups $\left(\bar{x}_{ \pm \mathrm{s}}\right)$

\begin{tabular}{lcccccc}
\hline Groups & Cases & Age (years) & Weight $(\mathrm{kg})$ & Courses (year) & Birth (s) & Number of weeks of pregnancy \\
\hline Experimental group & 32 & $60.03 \pm 2.24$ & $60.36 \pm 3.97$ & $3.19 \pm 0.97$ & $1.47 \pm 0.72$ & $39.56 \pm 1.01$ \\
Control group & 32 & $60.25 \pm 2.11$ & $60.08 \pm 4.17$ & $3.25 \pm 1.11$ & $1.50 \pm 0.67$ & $39.66 \pm 1.04$ \\
\hline
\end{tabular}

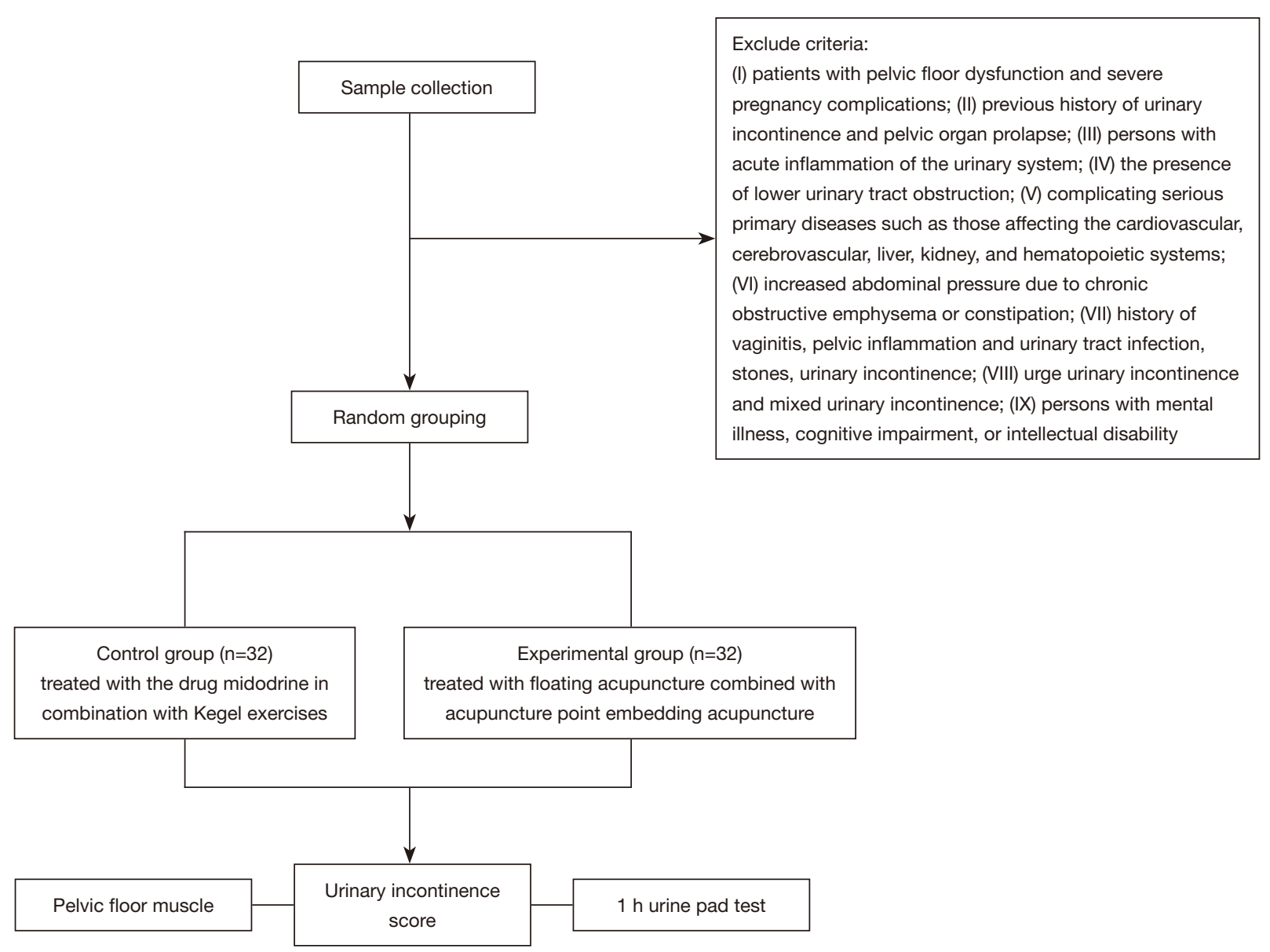

Figure 1 The Consolidated Standards of Reporting Trials (CONSORT) flow diagram of study participants.

and variance were expressed as mean \pm standard deviation $(\bar{x} \pm s)$. The inter-group measurement data were tested by independent sample t-test and the intra-group measurement data by paired sample t-test. Count data were tested by chisquare $\left(\chi^{2}\right)$. A difference was considered significant at $\mathrm{P}<0.05$.

\section{Results}

\section{General information}

Age, body weight, course of disease, number of births, and gestational weeks were not statistically significant between the 2 groups $(\mathrm{P}>0.05)$ (Table 1). The Consolidated Standards of Reporting Trials (CONSORT) diagram is shown in Figure 1.

\section{Comparison of pelvic floor muscle strength}

Before treatment, the pelvic floor muscle strength of the 2 groups was not statistically significant $(\mathrm{P}>0.05)$. The composition ratio of participants with pelvic floor muscle strength of the experimental group was significantly lower than that of the control group $(\mathrm{P}<0.001)$, and the composition ratio of participants with grade $4-5$ was higher 
Table 2 Comparison of pelvic floor muscle strength between 2 groups before and after treatment [cases (\%)]

\begin{tabular}{|c|c|c|c|c|c|c|c|c|}
\hline Groups & Cases & Time & Grade $0, \mathrm{n}(\%)$ & Grade 1, n (\%) & Grade 2, n (\%) & Grade 3, n (\%) & Grade 4, n (\%) & Grade 5, n (\%) \\
\hline Experimental group & & After treatment & 0 & $2(6.24)$ & $5(15.62)$ & $7(21.88)$ & $9(28.13)$ & $9(28.13)$ \\
\hline \multirow[t]{2}{*}{ Control group } & 32 & Before treatment & $11(34.38)$ & $17(53.12)$ & $4(12.50)$ & 0 & 0 & 0 \\
\hline & & After treatment & 0 & $14(43.74)$ & 7 (21.88) & $7(21.88)$ & $4(12.50)$ & 0 \\
\hline
\end{tabular}

Table 3 Comparison of IC-IQ-SF scores between the 2 groups before and after treatment $(\bar{x} \pm \mathrm{s})$

\begin{tabular}{lcccccc}
\hline Groups & Cases & Time & Frequency of leakage & Urine leakage & Impact on quality of life & Total scores \\
\hline Experimental group & 32 & Before treatment & $3.09 \pm 0.69$ & $3.84 \pm 0.81$ & $7.44 \pm 1.39$ & $14.38 \pm 2.14$ \\
& & After treatment & $2.19 \pm 0.59^{\star \star \star \# \#}$ & $2.50 \pm 0.72^{\star \star \star \#}$ & $5.69 \pm 1.15^{\star \star \star \#}$ & $10.38 \pm 1.72^{\star \star \star \# \# \#}$ \\
Control group & 32 & Before treatment & $3.03 \pm 0.82$ & $3.91 \pm 0.78$ & $7.44 \pm 1.44$ & $14.38 \pm 1.77$ \\
& & After treatment & $2.63 \pm 0.55^{\star}$ & $3.00 \pm 0.67^{\star \star \star}$ & $6.38 \pm 1.10^{\star \star}$ & $12.00 \pm 1.61^{\star \star \star}$ \\
\hline
\end{tabular}

After treatment, compared with before treatment, ${ }^{*} \mathrm{P}<0.05$, ${ }^{\star \star} \mathrm{P}<0.01$, ${ }^{\star \star \star} \mathrm{P}<0.001$; after treatment, compared with the control group, ${ }^{\#} \mathrm{P}<0.05,{ }^{\# \#} \mathrm{P}<0.01,{ }^{\# \# \#} \mathrm{P}<0.001$. IC-IQ-SF, international urinary incontinence advisory board urinary incontinence questionnaire.

Table 4 Comparison of leakage in the urine pad test before and after treatment $\left(\bar{x}_{ \pm}\right)$

\begin{tabular}{lccc}
\hline Groups & Cases & Time & Urine leakage $(\mathrm{g})$ \\
\hline Experimental group & 32 & Before treatment & $7.41 \pm 1.39$ \\
& & After treatment & $4.69 \pm 1.33^{* * * \#}$ \\
Control group & 32 & Before treatment & $7.38 \pm 1.76$ \\
& & After treatment & $5.91 \pm 1.53^{* * *}$ \\
\hline
\end{tabular}

After treatment, compared with before treatment, ${ }^{* * *} \mathrm{P}<0.001$; after treatment, compared with the control group, ${ }^{\# \#} \mathrm{P}<0.01$.

than that of the control group $(\mathrm{P}<0.001)$ (Table 2).

\section{IC-IQ-SF score comparison}

Before treatment, there was no statistically significant ICIQ-SF score between the 2 groups $(\mathrm{P}>0.05)$. After treatment, the frequency of urine leakage, the amount of urine leakage, the impact on the quality of life and the total score of the experimental group and the control group were lower than before treatment $(\mathrm{P}<0.001, \mathrm{P}<0.01, \mathrm{P}<0.05)$; After treatment, the difference between experimental group and control group was significant $(\mathrm{P}<0.01, \mathrm{P}<0.05, \mathrm{P}<0.001)$ (Table 3).

\section{Comparison of urinary leakage}

There was no significant difference in urinary leakage between the 2 groups before treatment $(P>0.05)$. The volume of urine leakage of the experimental group and the control group were significantly lower than those before treatment $(\mathrm{P}<0.001)$, and the difference between the experimental group and the control group was significant $(\mathrm{P}<0.01)$ (Table 4).

\section{Comparison of clinical effects}

The total effective rate of the experimental group was $90.63 \%$ (27/32) after 3 months of treatment, which was significantly higher than that of the control group $(71.88 \%$, 23/32) (Table 5).

\section{Discussion}

Postpartum SUI is common among middle-aged and elderly women (13-16). It is divided into three grades 
Table 5 Comparison of clinical effects between 2 groups

\begin{tabular}{lccccc}
\hline Groups & Cases & Cure, $\mathrm{n}(\%)$ & Effective cases, $\mathrm{n}(\%)$ & Ineffective cases, $\mathrm{n}(\%)$ & Total ineffective cases, $\mathrm{n}(\%)$ \\
\hline Experimental group & 32 & $22(68.75)$ & $7(21.88)$ & $3(9.37)$ & $27(90.63)$ \\
Control group & 32 & $15(46.88)$ & $8(25.00)$ & $9(28.12)$ & $23(71.88)$ \\
\hline
\end{tabular}

according to the severity of the disease. The first level is that urinary incontinence occurs only under severe stress (such as coughing, sneezing, and lifting heavy objects); the second level is that urinary incontinence occurs as long as it is under mild pressure when walking, standing, or shopping; the third level is the phenomenon of urinary incontinence regardless of any activity or posture. The gradual aging of the body, degenerative changes of pelvic floor muscular support structures, and decreased bladder sphincter SUI not only seriously physically affect the quality of life and quality of sexual life of the women affected, but can also cause anxiety and other negative emotions, which is not conducive to optimal postpartum recovery. Therefore, it is necessary to place adequate focus on the identification and treatment of SUI $(17,18)$. Currently, for mild and moderate SUI patients, non-surgical Kegel exercises are employed to reduce the severity (19) of urinary incontinence symptoms to some extent by strengthening and toning the pelvic floor muscles. However, due to the lengthy commitment period required by Kegel exercise treatment, there have been problems with patient adherence, which has resulted in poor treatment outcomes.

Previous studies have been conducted involving the treatment of SUI with floating acupuncture combined with acupuncture point embedding $(20,21)$. Floating acupuncture therapy was inspired by traditional acupuncture theory such as the floating thorn and burr technique mentioned in the Huangdi Neijing (Yellow Emperor's Classic of Internal Medicine), yet it is different from traditional acupuncture therapy. According to the theory of affected muscle and MTrP, it is considered that muscle and fascia tissue do not exist in isolation, but interact with each other (22). As a result, the affected muscles of SUI patients are not limited to the bottom of the pelvis, but also appear in the lower abdomen and the medial side of the lower extremity, such as the lower segment of the rectus abdominis, the oblique abdominal muscle, adductor muscle group of the thigh, and the medial group of the quadriceps femoris in this study. During the treatment, the affected muscles are detected through palpation of the lower abdomen and lower extremities, then the affected muscles are repeatedly contracted and relaxed by acupuncture and reperfusion activities to accelerate the velocity of blood flow (23). This is a special method of acupuncture, and its theoretical basis can be traced back to the shallow prick method of the theory of 12 skin areas in the Huangdi Neijing. When SUI is the result of pregnancy, labor, qi and blood consumption, kidney qi damage, and subsequently bladder and urinary incontinence, the disease pathogenesis combined with modern anatomical knowledge will indicate that acupuncture treatment is focused on the foot Taiyang bladder meridian, Ren Mai meridian, and foot Shaoyin kidney meridian points, acupoints which are mostly distributed in the lower abdomen and lumbosacral region. The acupoint Zhongji (CV3) belongs to the Ren Mai meridian, is a meeting point of the Zusanyin and Ren Mai meridians, and is a bladder collection point; Guanyuan (CV4) point is on the Ren Mai channel, is also a meeting point of the Zusanyin and Renmai meridians, and is the small intestine collection point, from where the loss of vital energy can be restored, and is clinical used for genitourinary diseases. After embedding the needle into the Zhongji and Guanyuan acupoints, through continuous and stable stimulation of the skin and collaterals, the meridian qi and blood flow becomes smooth, and the function of the meridians and viscera are adjusted to achieve the purpose of treating SUI. Notably, the subcutaneously embedded needle will not have the problems of stuck needle, halo needle (during the acupuncture process, the patient suddenly experienced dizziness, dizziness, palpitation, nausea, and even fainting), and so on, and will not affect the normal life of patients, so the acceptance of this technique should be better than that of traditional acupuncture. The treatment of floating needle therapy combined with acupuncture point needle embedding can release the affected muscle quickly, relieving the pelvic floor muscle and strengthening the muscle tone, thus restoring the function of the urethral support system and improving the condition of urinary leakage.

According to the results of this study, the composition ratio of participants with pelvic floor muscle strength assessed as $0-3$ in the experimental group was significantly 
lower than that in the control group, while that of participants with grade 4-5 was significantly higher than that in the control group. It is suggested that floating needle therapy combined with acupuncture point embedding can effectively increase pelvic floor muscle strength in SUI patients. Pelvic floor muscle is composed of the urethral sphincter, anal sphincter, levator ani muscle, and other rhabdomyoid muscles. Laxity of the pelvic floor muscle can cause the bladder neck to droop and leak urine when abdominal pressure increases, resulting in SUI. Therefore, restoring pelvic floor muscle function and enhancing detrusor group stability are the main measures to treat SUI (24-26). Moreover, the IC-IQ-SF score of the experimental group after treatment and the amount of urine leakage in the $1 \mathrm{~h}$ pad test were smaller than those of the control group before treatment and after treatment, suggesting that floating needle therapy combined with acupuncture point embedding therapy can effectively reduce the degree of urinary incontinence and have a significant effect on SUI.

In summary, floating needle therapy combined with acupoint needle embedding increased pelvic floor muscle strength and improved urinary incontinence, and the total effective rate was 90.63 , which was higher than that of the control group. The curative effect was remarkable and is worthy of clinical promotion.

\section{Acknowledgments}

Funding: This research was supported by the Zhejiang Traditional Chinese Medicine Science and Technology Plan (2021ZA126).

\section{Footnote}

Reporting Checklist: The authors have completed the CONSORT reporting checklist. Available at https://dx.doi. org/10.21037/apm-21-1382

Trial Protocol: Available at https://dx.doi.org/10.21037/apm21-1382

Data Sharing Statement: Available at https://dx.doi. org/10.21037/apm-21-1382

Conflicts of Interest: All authors have completed the ICMJE uniform disclosure form (available at https://dx.doi. org/10.21037/apm-21-1382). The authors have no conflicts of interest to declare.
Ethical Statement: The authors are accountable for all aspects of the work in ensuring that questions related to the accuracy or integrity of any part of the work are appropriately investigated and resolved. All procedures performed in this study involving human participants were in accordance with the Declaration of Helsinki (as revised in 2013). The study was approved by the Ethics Committee of Ningbo Municipal Hospital of TCM Affiliated Hospital of Zhejiang Chinese Medicine University (AF/SG0110.1/20201215005) and informed consent was provided by all participants.

Open Access Statement: This is an Open Access article distributed in accordance with the Creative Commons Attribution-NonCommercial-NoDerivs 4.0 International License (CC BY-NC-ND 4.0), which permits the noncommercial replication and distribution of the article with the strict proviso that no changes or edits are made and the original work is properly cited (including links to both the formal publication through the relevant DOI and the license). See: https://creativecommons.org/licenses/by-nc-nd/4.0/.

\section{References}

1. Falah-Hassani K, Reeves J, Shiri R, et al. The pathophysiology of stress urinary incontinence: a systematic review and meta-analysis. Int Urogynecol J 2021;32:501-52.

2. Tricard T, Al Hashimi I, Schroeder A, et al. Real-life outcomes after artificial urinary sphincter explantation in women suffering from severe stress incontinence. World J Urol 2021. [Epub ahead of print]. doi: 10.1007/s00345021-03672-y.

3. Løwenstein E, Jepsen R, Andersen L L, et al. Prevalence of urinary incontinence among women with diabetes in the Lolland-Falster Health Study, Denmark. Neurourol Urodyn 2021;40:855-67.

4. Luginbuehl H, Lehmann C, Koenig I, et al. Involuntary reflexive pelvic floor muscle training in addition to standard training versus standard training alone for women with stress urinary incontinence: a randomized controlled trial. Int Urogynecol J 2021. [Epub ahead of print]. doi: 10.1007/s00192-021-04701-5.

5. Gonzales AL, Barnes KL, Qualls CR, et al. Prevalence and Treatment of Postpartum Stress Urinary Incontinence: A Systematic Review. Female Pelvic Med Reconstr Surg 2021;27:e139-45.

6. Khayyami Y, Elmelund M, Klarskov N. Urinary 
incontinence before and after pelvic organ prolapse surgery-A national database study. Int Urogynecol J 2021. [Epub ahead of print]. doi: 10.1007/s00192-021-04738-6.

7. Shen L, Yang J, Bai X, Sun Z. Analysis of the current status of pelvic floor dysfunction in urban women in Xi'an City. Ann Palliat Med 2020;9:979-84.

8. Yang J, Cheng J W, Wagner H, et al. The effect of high impact crossfit exercises on stress urinary incontinence in physically active women. Neurourol Urodyn 2019;38:749-56.

9. Yang N, Ge X, Ye J, et al. Efficacy of acupuncture for urinary incontinence in middle-aged and elderly women: A systematic review and meta-analysis of randomized controlled trials. Eur J Obstet Gynecol Reprod Biol 2021;257:138-43.

10. Bezier E. Impact of biofeedback probes used in the selfrehabilitation of pelvic floor muscles in women with stress urinary incontinence. Literature review. Prog Urol 2021.

11. Baron M, Le Normand L, Paret F, et al. Management of post-operative urinary incontinence after midurethral sling explantation for pelvic or perineal pain. Int Urogynecol J 2021. [Epub ahead of print]. doi: 10.1007/s00192-021-04759-1.

12. Berger AA, Tan-Kim J, Menefee SA. The impact of midurethral sling surgery on the development of urinary tract infections. Int Urogynecol J 2021. [Epub ahead of print]. doi: 10.1007/s00192-021-04779-x.

13. Li T, Zhang YJ, Zhang HL, et al. Prevalence and Risk Factors of Stress Urinary Incontinence Among Perimenopausal Women and Its Influence on Daily Life in Women with Sexual Desire Problem. Curr Med Sci 2019;39:615-21.

14. Geng L, Zheng Y, Zhou Y, et al. The prevalence and determinants of genitourinary syndrome of menopause in Chinese mid-life women: a single-center study. Climacteric 2018;21:478-82.

15. Kuprasertkul A, Christie AL, Zimmern P. A standardized telephone interview with validated questionnaires for very long-term evaluation of women lost to follow-up after a stress urinary incontinence procedure. Low Urin Tract Symptoms 2021. [Epub ahead of print]. doi: 10.1111/ luts. 12378 .

16. Lee U J, Feinstein L, Ward J B, et al. Prevalence of Urinary Incontinence among a Nationally Representative Sample of Women, 2005-2016: Findings from the Urologic Diseases in America Project. J Urol 2021;205:1718-24.

17. Desai SA, Vakil Z, Kroumpouzos G. Transcutaneous Temperature-Controlled Radiofrequency Treatment: Improvement in Female Genital Appearance, Sexual
Dysfunction, and Stress Urinary Incontinence. Aesthet Surg J 2021. [Epub ahead of print]. doi: 10.1093/asj/sjab174.

18. Abufaraj M, Xu T, Cao C, et al. Prevalence and Trends in Urinary Incontinence Among Women in the United States 2005-2018. Am J Obstet Gynecol 2021. [Epub ahead of print]. doi: 10.1016/j.ajog.2021.03.016.

19. Nilsen I, Rebolledo G, Acharya G, et al. Mechanical oscillations superimposed on the pelvic floor muscles during Kegel exercises reduce urine leakage in women suffering from stress urinary incontinence: A prospective cohort study with a 2-year follow up. Acta Obstet Gynecol Scand 2018;97:1185-91.

20. Yang J, Cheng Y, Zhao L, et al. Acupuncture and related therapies for stress urinary incontinence: A protocol for systematic review and network meta-analysis. Medicine (Baltimore) 2020;99:e21033.

21. Liu Z, Liu Y, Liu B. Acupuncture for Stress Urinary Incontinence-Reply. JAMA 2017;318:1500.

22. Zhong M, Zhang X. FlU Zhonghua's clinical experience of Fu's subcutaneous needling for cervical spondylosis. Zhongguo Zhen Jiu 2015;35:823-6.

23. Liu Z, Liu Y, Xu H, et al. Effect of Electroacupuncture on Urinary Leakage Among Women With Stress Urinary Incontinence: A Randomized Clinical Trial. JAMA 2017;317:2493-501.

24. Fontenele MQS, Moreira MA, de Moura A, et al. Pelvic floor dysfunction distress is correlated with quality of life, but not with muscle function. Arch Gynecol Obstet 2021;303:143-9.

25. Mclean L, Charette M, Varette K, et al. Pelvic floor muscle training as an adjunct to a midurethral sling: a single-blind randomised controlled trial. Int Urogynecol J 2021. [Epub ahead of print]. doi: 10.1007/s00192-020-04668-9.

26. Hwang UJ, Lee MS, Jung SH, et al. Relationship Between Sexual Function and Pelvic Floor and Hip Muscle Strength in Women With Stress Urinary Incontinence. Sex Med 2021;9:100325.

(English Language Editor: J. Jones)

Cite this article as: Chen $\mathrm{F}$, Zhou $\mathrm{J}, \mathrm{Wu}$ W, Qian X. Study on the therapeutic effect of floating needle therapy combined with pressing acupoint embedding for female stress urinary incontinence after childbirth: a randomized trial. Ann Palliat Med 2021;10(7):7786-7793. doi: 10.21037/apm-21-1382 